\title{
Cefquinome Controlled Size Submicron Particles Precipitation by SEDS Process Using Annular Gap Nozzle
}

\author{
Kefeng Xiao, ${ }^{1,2,3}$ Weiqiang Wang, ${ }^{1,3}$ Dedong $\mathrm{Hu},{ }^{3,4}$ Yanpeng $\mathrm{Qu},{ }^{1,3}$ \\ Zhihui Hao, ${ }^{2}$ and Leilei Wang ${ }^{2}$ \\ ${ }^{1}$ School of Mechanical Engineering, Shandong University, Jinan 250061, China \\ ${ }^{2}$ College of Chemistry and Pharmaceutical Science, Qingdao Agricultural University, Qingdao 266109, China \\ ${ }^{3}$ Shanda-Lunan Research Institute of Supercritical Fluid Technology, Shandong University, Jinan 250061, China \\ ${ }^{4}$ College of Electromechanical Engineering, Qingdao University of Science and Technology, Qingdao 266061, China
}

Correspondence should be addressed to Weiqiang Wang; wqwang@sdu.edu.cn

Received 23 March 2017; Accepted 30 May 2017; Published 3 July 2017

Academic Editor: Jerzy Baldyga

Copyright (c) 2017 Kefeng Xiao et al. This is an open access article distributed under the Creative Commons Attribution License, which permits unrestricted use, distribution, and reproduction in any medium, provided the original work is properly cited.

\begin{abstract}
An annular gap nozzle was applied in solution enhanced dispersion by supercritical fluids (SEDS) process to prepare cefquinome controlled size submicron particles so as to enhance their efficacy. Analysis results of orthogonal experiments indicated that the concentration of solution was the primary factor to affect particle sizes in SEDS process, and feeding speed of solution, precipitation pressure, and precipitation temperature ranked second to fourth. Meanwhile, the optimal operating conditions were that solution concentration was $100 \mathrm{mg} / \mathrm{mL}$, feeding speed was $9 \mathrm{~mL} / \mathrm{min}$, precipitation pressure was $10 \mathrm{MPa}$, and precipitation temperature was $316 \mathrm{~K}$. The confirmatory experiment showed that $D_{50}$ of processed cefquinome particles in optimal operating conditions was $0.73 \mu \mathrm{m}$. Moreover, univariate effect analysis showed that the cefquinome particle size increased with the increase of concentration of the solution or precipitation pressure but decreased with the increase of solution feeding speed. When precipitation temperature increased, the cefquinome particle size showed highest point. Moreover, characterization of processed cefquinome particles was analyzed by SEM, FT-IR, and XRD. Analysis results indicated that the surface appearance of processed cefquinome particles was flakes. The chemical structure of processed cefquinome particles was not changed, and the crystallinity of processed cefquinome particles was a little lower than that of raw cefquinome particles.
\end{abstract}

\section{Introduction}

Cefquinome is the fourth-generation cephalosporin antibiotics for animal and is a broad-spectrum $\beta$-lactam antibiotic in the treatment of clinical mastitis. Cefquinome is licensed as a combination therapy for E. coli mastitis in UK [1] and is used widely for respiratory disease of pigs, horses sepsis, and cows udder inflammation, respiratory tract infections, and other serious infections caused by susceptible strains in swine and cattle [2]. The mean particle diameter of raw cefquinome is about 2 microns. The particle size of the drug affects absorption rate and bioavailability either by topical or by injectable applications [3]. The drug has to be formulated into uniform microparticles in appropriate size and shape for the purpose of achieving optimal efficiency of therapy [4]. Ostwald-Freundlich equation indicates that when mean particle diameter of drug is less than 1 micron, its solubility would increase greatly and thereby enhance absorption rate and drug bioavailability [5]. Therefore, it is urgent to study how to reduce the particle size and prepare cefquinome controlled size submicron particles.

In pharmaceutical field, several technologies, including spray drying [6], antisolvent method [7], emulsification method [8], and supercritical fluid (SCF) process $[9,10]$, are used to control particle size. Among these methods, SCF process has absorbed more and more attention because of no solvent residue, low operating temperature, and controllable process [11]. Solution enhanced dispersion by supercritical fluids (SEDS) process is an important branch method of SCF process [12]. SEDS process can deal with the drug insoluble in water and control easily the particle size of drug [13]. 


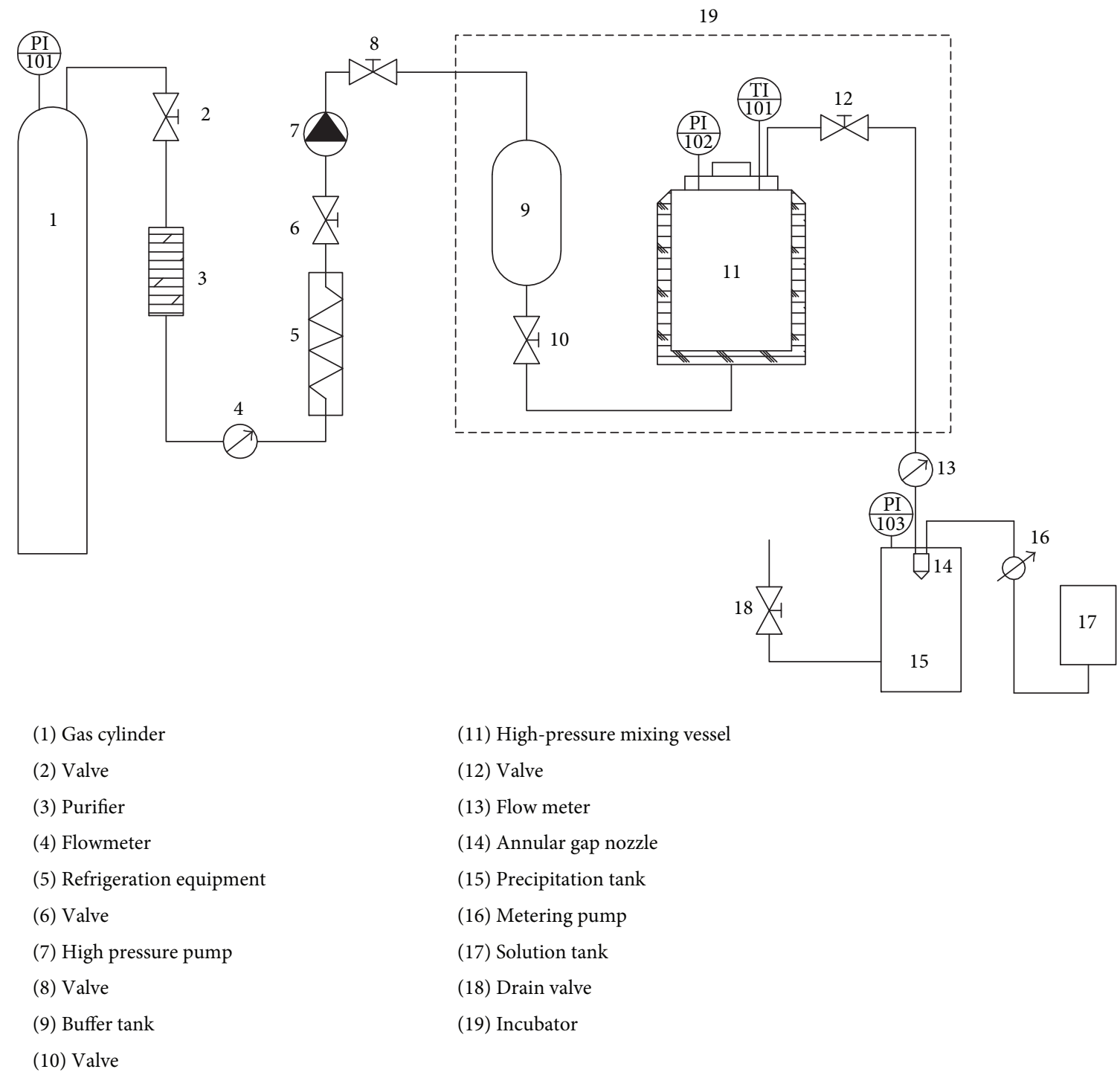

FIGURE 1: The flow diagram of the SEDS process.

It has been suggested that jet breakup in spray process is a controlling factor in SEDS process [14]. The nozzle becomes the key component to adjust jet breakup in SEDS process. Many nozzles have been applied in SEDS process, such as coaxial nozzles [15], internal twin-fluid mixing nozzles [16], jet-swirl nozzle [17], four-pinhole nozzle [18], prefilming nozzle [19], and annulus gap nozzle [20]. We have studied preparing cefquinome particles in SEDS process by using four-pinhole nozzle and obtained controlled size submicron particles [18]. However, there still existed some problems such as easy to block and small processing capacity. In order to solve these problems, in this work, we study preparing cefquinome particles in SEDS process by using a new kind of annular gap nozzle.

\section{Materials and Methods}

2.1. Materials. Cefquinome (mass fraction purity $>82 \%$, calculated by cefquinome content) was purchased from Qilu
ShengHua Pharmaceutical Co., Ltd. Carbon dioxide (purity $>$ 99\%), acted as antisolvent, was supplied by Jinan German Foreign Specialty Gases Co., Ltd. Dimethyl sulfoxide (DMSO) (analytical grade), acted as solvent of cefquinome, was supplied by Tianjin Fuyu Fine Chemical Co., Ltd.

2.2. Equipment and Operating Procedure. Semicontinuous SEDS process equipment (HKY-3, Nantong Huaan Supercritical Extraction Co., Ltd., China) was employed. The flow diagram was shown in Figure 1. The detail operating process was described in our previous work [18]. In brief, carbon dioxide was purified and liquefied firstly and continuously introduced into the precipitation tank through an annular gap nozzle. The temperature of carbon dioxide can be adjusted by adjusting the temperature of the incubator. The pressure in precipitation tank was controlled by adjusting valve 18 . When the pressure and temperature reached desired values, pure DMSO was continuously introduced into the 


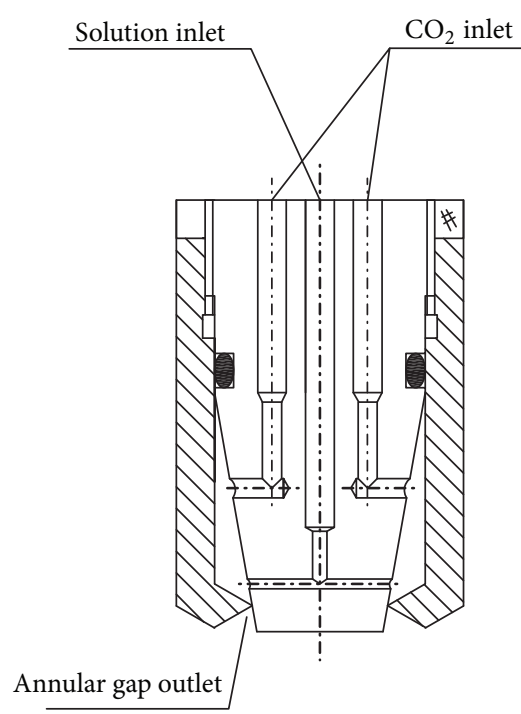

(a) Internal structure

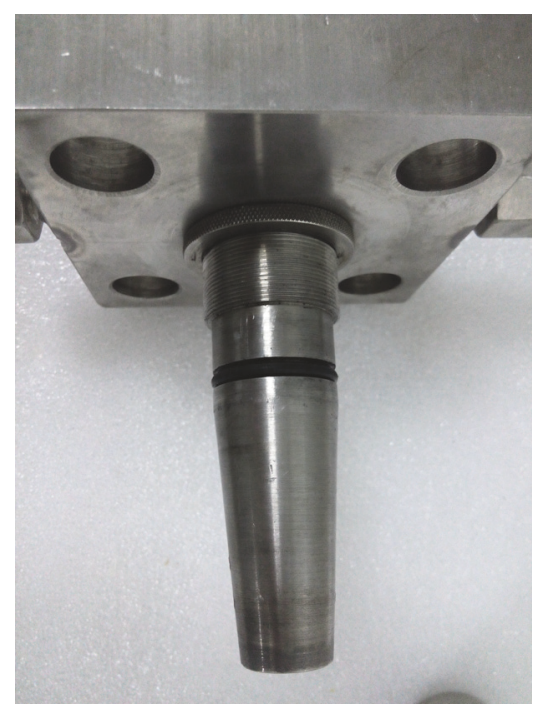

(b) Surface appearance of real annular gap nozzle

Figure 2: The structure of annular gap nozzle.

precipitation tank by metering pump through the annular gap nozzle until the pressure and temperature were resteady. Then, cefquinome/DMSO solution was pumped into the annular gap nozzle and then sprayed out to produce the cefquinome precipitation. After injection of the solution was finished, supercritical carbon dioxide continued to be pumped into the nozzle for forty minutes so that all remaining DMSO was carried out of the precipitation tank. Then, the precipitation tank was depressurized gradually to atmospheric pressure. Finally, the processed particles were collected from the bottom of the precipitation tank for further characterization analysis. Annular gap nozzle was the key component of the SEDS process equipment. The structure of the nozzle was shown in Figure 2. The annular gap nozzle was designed especially for SEDS process. Supercritical $\mathrm{CO}_{2}$ and cefquinome/DMSO solution were introduced separately from different channels, then mixed, and then sprayed out quickly. The distance of the annular gap can adjust according to different requirements. In this study, the distance of the annular gap was $0.2 \mathrm{~mm}$.

In order to study the optimal operating conditions of preparing cefquinome controlled size submicron particles by SEDS process, an orthogonal $L_{9}\left(3^{4}\right)$ test design was employed. Cefquinome concentration, feeding speed of solution, precipitation pressure, and temperature were selected as the factors of the experiment, and the level range of each factor was determined as shown in Table 1 . All of these were selected based on the results of preliminary experiments and related study $[18,19,21]$. The median diameter $\left(D_{50}\right)$ of particles acted as the dependent variable changing with these factors. Design Expert 8.0 software was used to analyze experimental results according to range analysis principle.

\subsection{Characterization Methods}

2.3.1. Particle Size Measurement. The size of cefquinome particles was measured by a laser particle size analyzer (BT9300H, Dandong Baxter Instrument Co., Ltd., China). Before measurement, the cefquinome particles were suspended in ultrapure water, and then the suspension was stirred in an ultrasonic disperser for 5 minutes so as to disperse effectively. Background test was conducted in a clean sample cell only filled with ultrapure water. Then the suspension dispersed well was injected into the sample cell. Finally, the particle sizes, expressed by the median diameter $\left(D_{50}\right)$, were obtained. Each measurement was repeated three times.

2.3.2. Surface Morphology Analysis. The surface morphologies of cefquinome particles were observed by a scanning electron microscope (SEM) (NOVA NANOSEM 450, American FEI Corporation, USA). Before observation, Cefquinome particles were stuck on the test bench with double-sided adhesive carbon tape and then were coated with a thin layer of gold under high vacuum conditions.

2.3.3. FT-IR Spectroscopy. Fourier transform infrared (FTIR) spectra were recorded on a FT-IR Spectrometer (Nicolet IR200, Thermo Fisher Scientific, Inc., USA) with a resolution of $4 \mathrm{~cm}^{-1}$ at room temperature. The scanning range was $400-4000 \mathrm{~cm}^{-1}$. Cefquinome particles were dispersed in $\mathrm{KBr}$ and the mixture was pressed into disc form. A pellet was formed including $2.5 \mathrm{mg}$ cefquinome particles and $250 \mathrm{mg}$ $\mathrm{KBr}$. Data was analyzed by Omnic.

2.3.4. XRD Analysis. The crystalline structure of the samples was analyzed by an X-ray powder diffractometer (D8 ADVANCE, Bruker AXS, German) with $\mathrm{Cu} \mathrm{K} \alpha$ radiation at 
TABLE 1: Factors and levels of the orthogonal array design.

\begin{tabular}{lcccc}
\hline Factor & $\begin{array}{c}\text { Concentration of solution } \\
(\mathrm{mg} / \mathrm{mL})\end{array}$ & $\begin{array}{c}\text { Solution feeding speed } \\
(\mathrm{mL} / \mathrm{min})\end{array}$ & Precipitation pressure (MPa) & Precipitation temperature (K) \\
\hline 1 & 100 & 3 & 10 & 306 \\
2 & 200 & 6 & 13 & 311 \\
3 & 300 & 9 & 16 & 316 \\
\hline
\end{tabular}

$40 \mathrm{kV}$ and $40 \mathrm{~mA}$. The samples were scanned between $10^{\circ}$ and $80^{\circ}(2 \theta)$.

\section{Results and Discussion}

3.1. Optimization Study. The SEDS process is a very complex process. When precipitation occurs, nucleation occurs simultaneously and then particles grow fastly accompanied with aggregation and breakage [22]. The occurrence and development of these processes affect greatly the particle size, appearance, and even crystal form, whereas these processes can be influenced by the change of operating parameters, such as solution flow rate, solution concentration, pressure and temperature.

The analyzing results of the experiments were shown in Table 2. According to range analysis principle, the greater the range is, the greater the impact of the factor is. It indicated in Table 2 that the range of factor $A$ was the greatest in the four factors and the range of the four factors was sorted in descending order as follows: $A>B>C>D$. Therefore, the effect of solution concentration on particle size was the greatest, and the effect of solution feeding speed, precipitation pressure, and temperature ranked second to fourth. The order of these factors is nearly the same as that in [23], except that the order of temperature and pressure is slightly different. The best particle size obtained in the experiments was $0.80 \mu \mathrm{m}$. According to range analysis, the conditions of $A_{1} B_{3} C_{1} D_{3}$ were the optimal conditions; that is, the solution concentration was $100 \mathrm{mg} / \mathrm{mL}$, the feeding speed was $9 \mathrm{~mL} / \mathrm{min}$, precipitation pressure was $10 \mathrm{MPa}$, and precipitation temperature was $316 \mathrm{~K}$. In the optimal operating conditions, confirmatory test was conducted and $D_{50}$ of processed cefquinome particles was $0.73 \mu \mathrm{m}$.

3.2. Univariate Effect. The univariate effects on processed cefquinome particles size were shown in Figures 3(a)-3(d). $D_{50}$ was selected to indicate particle size. As shown in Figure 3(a), $D_{50}$ of processed cefquinome particles increased when the concentration of cefquinome increased from 100 to $300 \mathrm{mg} / \mathrm{mL}$. The trend of particle size change with solution concentration is consistent with that in $[5,24]$. In SEDS process, nucleation occurs in the nozzle, whereas growth completes in the precipitation tank [24]. With the increase of solution concentration, the surface tension between solution and supercritical carbon dioxide increases, Weber number decreases, and particle size increases [23]. In addition, after being introduced into precipitation tank, the solution in high concentration cannot be atomized well. Breaking time of the droplet increases so that the nucleation time of particles increases, leading to larger particle size.

As shown in Figure 3(b), $D_{50}$ decreased when the feeding speed of the solution increased from 3 to $9 \mathrm{~mL} / \mathrm{min}$. The increase of the flow rate makes the contact area between the supercritical fluid and the organic solution decrease, and the mass transfer effect is weakened, resulting in the decrease of the supersaturation rate [4]. The lower supersaturation reduces the amount of nucleation of the crystal, resulting in less agglomeration. Moreover, increase of the flow rate can exacerbate droplet breakage. All these reasons cause the prepared particles to have a smaller particle size.

In Figure 3(c), it indicated that $D_{50}$ increased with the increase of precipitation pressure. This trend is consistent with the trend in the literature [23] when the pressure is higher than $12 \mathrm{Mpa}$. With the increase of pressure, the diffusion coefficient and the diffusion rate of supercritical carbon dioxide increase. Moreover, with the increase of pressure, the density of supercritical carbon dioxide increases, and the contact area between the supercritical carbon dioxide and the organic solution increases. And then mass transfer effect increases, resulting in increased supersaturation rate. Therefore the possibility of a large number of nuclei forming large-size particle by growing and aggregating increases.

The effect of precipitation temperature on $D_{50}$ was complex, which can be seen from Figure $3(d) . D_{50}$ increased and then decreased when the precipitation temperature increased from $306 \mathrm{~K}$ to $316 \mathrm{~K}$. This trend is consistent with the trend in [5]. It is mainly because that when preparing drug particles in SEDS process, the effect of temperature on the particle size has two aspects. On the one hand, with the increase of temperature, the kinetic energy of the solvent molecules increases and the solvent diffusion rate increases. On the other hand, with the increase of temperature, the density of the supercritical carbon dioxide decreases, and the diffusion coefficient of the supercritical carbon dioxide decreases. The combined effect of these two aspects leads to the trend.

3.3. Surface Appearance Analysis. SEM image of raw cefquinome particles was shown in Figure 4. The surface appearance of raw cefquinome particles was irregular blocks. SEM image of processed cefquinome was shown in Figure 5. It indicated that these particles were flaky. Most of these particles aggregated together. When these particles were dispersed in superpure water by supersonic wave, they could be separated, which had been verified in measuring particle 


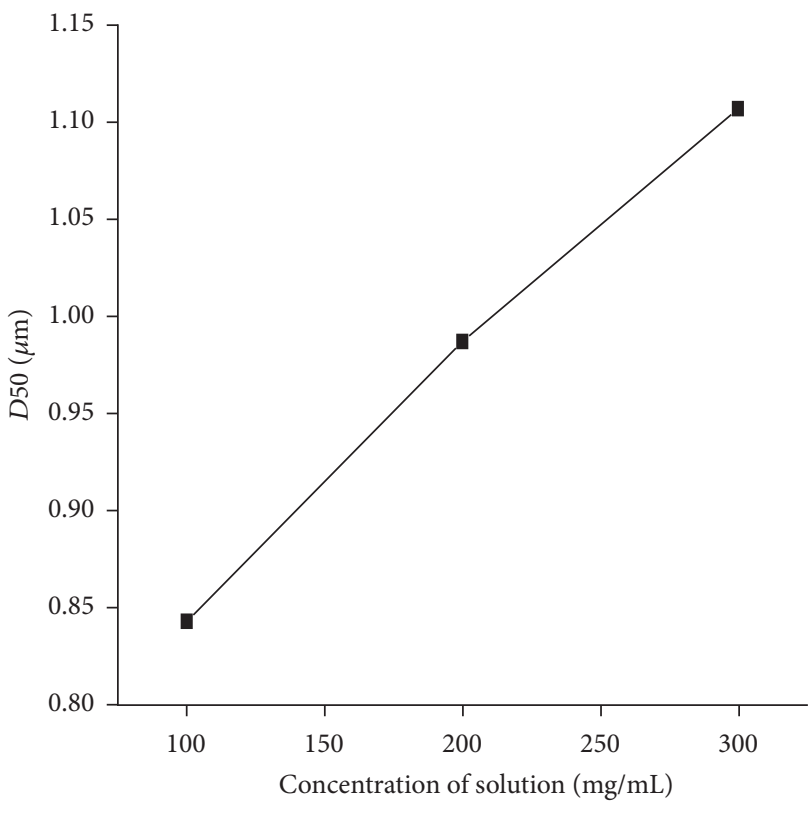

(a) A:Concentration of solution

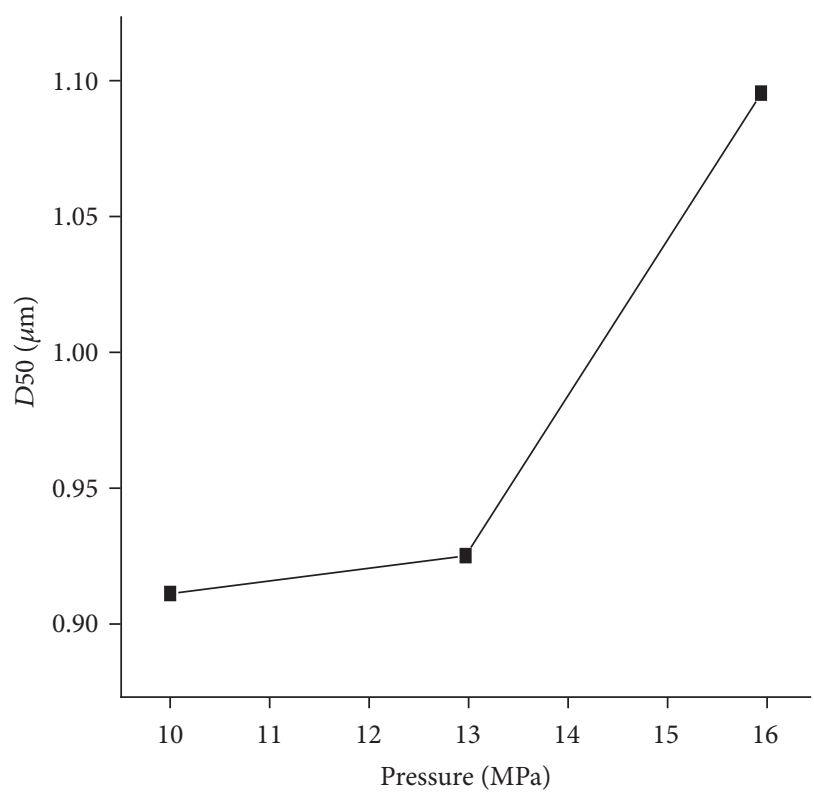

(c) C: Precipitation pressure

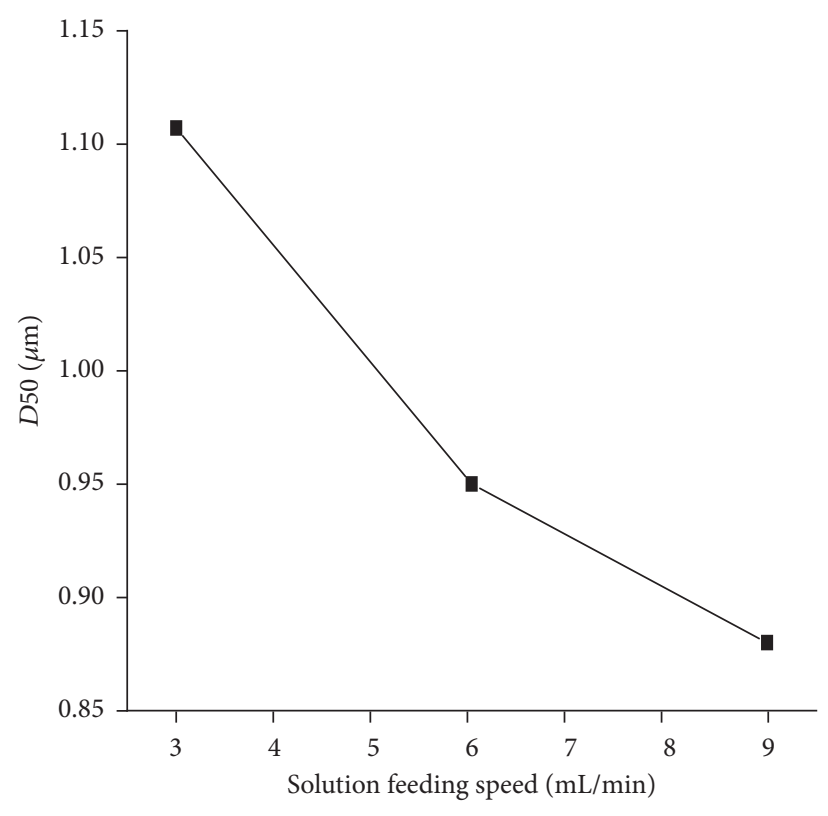

(b) B: Solution feeding speed

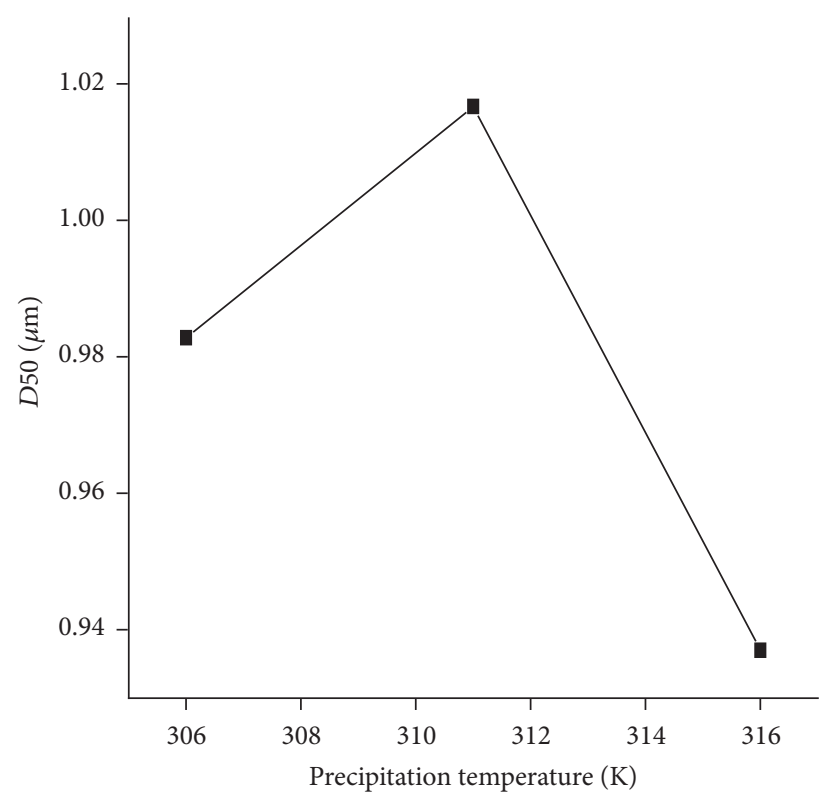

(d) D: Precipitation temperature

FIGURE 3: The univariate effects on processed cefquinome particles size.

size by laser particle size analyzer. In pharmaceutical industry, supersonic separation is a common process. Therefore, the agglomeration of the particles cannot affect the efficacy of cefquinome in practical pharmaceutical industry.

3.4. Result of FT-IR. FT-IR examinations were carried out to mainly obtain the changed information chemical structure of cefquinome after being processed by SEDS process. The FTIR spectra of raw cefquinome and processed cefquinome were shown in Figure 6. It indicated that the characteristic peaks of the two FT-IR spectra are almost the same. Therefore, the chemical structure of cefquinome does not change after being processed by SEDS process. It can be concluded that the SEDS process by using annular gap nozzle changed only particle size and surface morphology but not chemical structure.

3.5. Result of XRD. The XRD results of raw and processed cefquinome obtained in the study was shown in Figure 7. The characteristic high intensity diffraction peaks of raw cefquinome at the diffraction angles of $2 \theta=12.6^{\circ}, 16.1^{\circ}, 23.9^{\circ}$, and $24.6^{\circ}$ indicated the existence of its natural crystalline form. Whereas the processed cefquinome shows a diffraction 
TABLE 2: Analysis results of $L_{9}\left(3^{4}\right)$ test.

\begin{tabular}{|c|c|c|c|c|c|}
\hline Run & $\begin{array}{c}(A) \text { concentration of } \\
\text { solution }(\mathrm{mg} / \mathrm{mL})\end{array}$ & $\begin{array}{l}\text { (B) solution feeding } \\
\text { speed }(\mathrm{mL} / \mathrm{min})\end{array}$ & $\begin{array}{l}(C) \text { precipitation } \\
\text { pressure }(\mathrm{MPa})\end{array}$ & $\begin{array}{l}(D) \text { precipitation } \\
\text { temperature }\left({ }^{\circ} \mathrm{C}\right)\end{array}$ & $\begin{array}{l}\text { Average diameter } \\
(\mu \mathrm{m})\end{array}$ \\
\hline 1 & 1 & 1 & 1 & 1 & 0.91 \\
\hline 2 & 1 & 2 & 2 & 2 & 0.80 \\
\hline 3 & 1 & 3 & 3 & 3 & 0.82 \\
\hline 4 & 2 & 1 & 2 & 3 & 1.02 \\
\hline 5 & 2 & 2 & 3 & 1 & 1.08 \\
\hline 6 & 2 & 3 & 1 & 2 & 0.86 \\
\hline 7 & 3 & 1 & 3 & 2 & 1.39 \\
\hline 8 & 3 & 2 & 1 & 3 & 0.97 \\
\hline 9 & 3 & 3 & 2 & 1 & 0.96 \\
\hline$K 1^{\mathrm{a}}$ & 0.843 & 1.107 & 0.913 & 0.983 & \\
\hline$K 2$ & 0.987 & 0.95 & 0.927 & 1.017 & \\
\hline$K 3$ & 1.107 & 0.88 & 1.097 & 0.937 & \\
\hline$R^{\mathrm{b}}$ & 0.264 & 0.227 & 0.184 & 0.08 & \\
\hline Optimal level & $A 1$ & $B 3$ & $C 1$ & D3 & \\
\hline
\end{tabular}

${ }^{\mathrm{a}} K_{i}{ }^{A}=\Sigma\left(\right.$ mean particle size at $\left.A_{i}\right) / 3$, the mean values of mean particle size for a certain factor at each level with standard deviation.

${ }^{\mathrm{b}} R_{i}{ }^{A}=\max \left\{K_{i}{ }^{A}\right\}-\min \left\{K_{i}^{A}\right\}$.

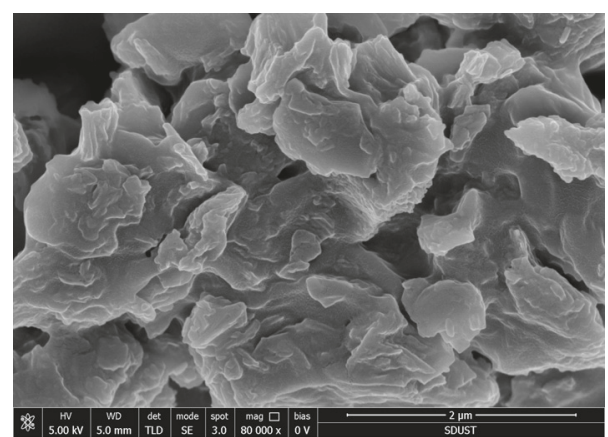

FIGURE 4: SEM image of raw cefquinome.

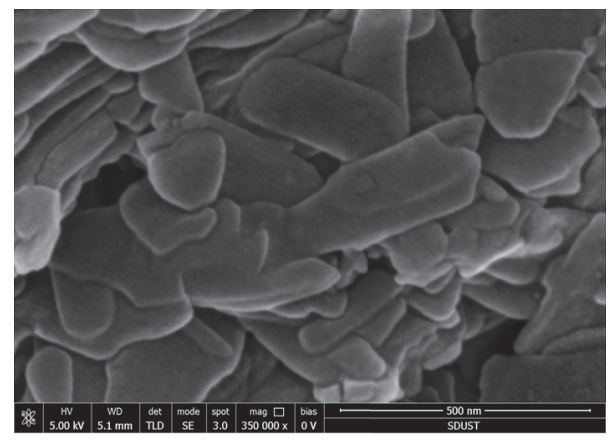

FIGURE 5: SEM image of processed cefquinome.

pattern with similar diffraction angles, the peak intensity is a little less than that of raw cefquinome. This indicates that cefquinome particles after being processed by SEDS
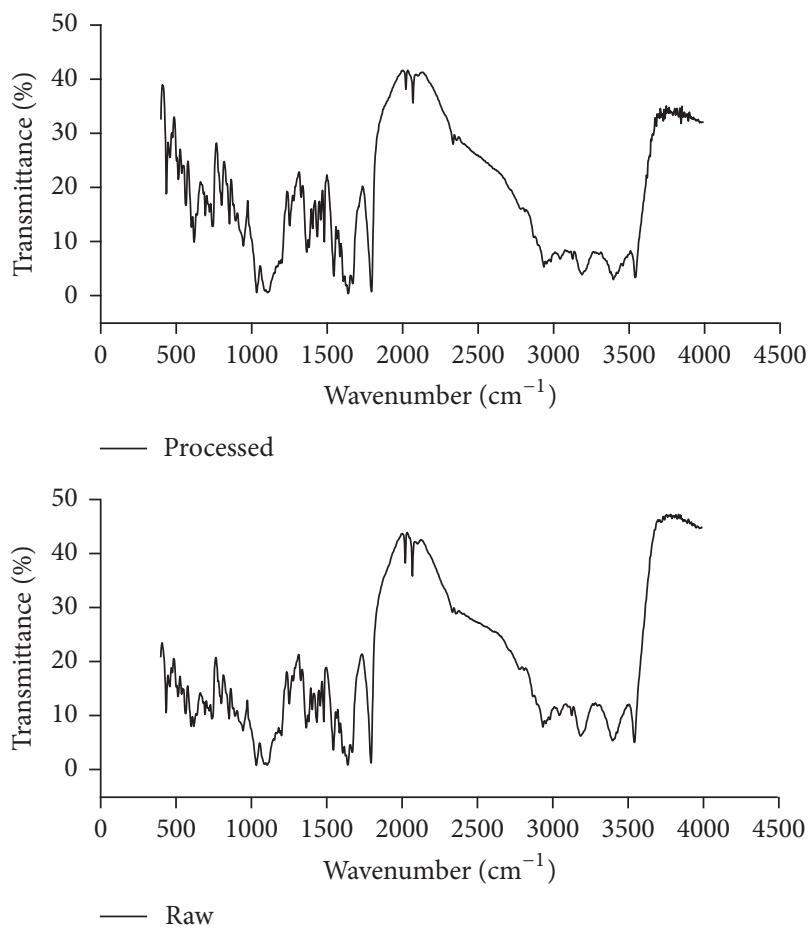

FIGURE 6: FT-IR spectra of raw cefquinome and processed cefquinome by SEDS process.

process are less crystalline. Therefore, it can be concluded that SEDS process using annular gap nozzle can decrease slightly the crystallinity of cefquinome particles. Low crystallinity is more helpful for enhancing dissolution rate or bioavailability than crystals [25]. 

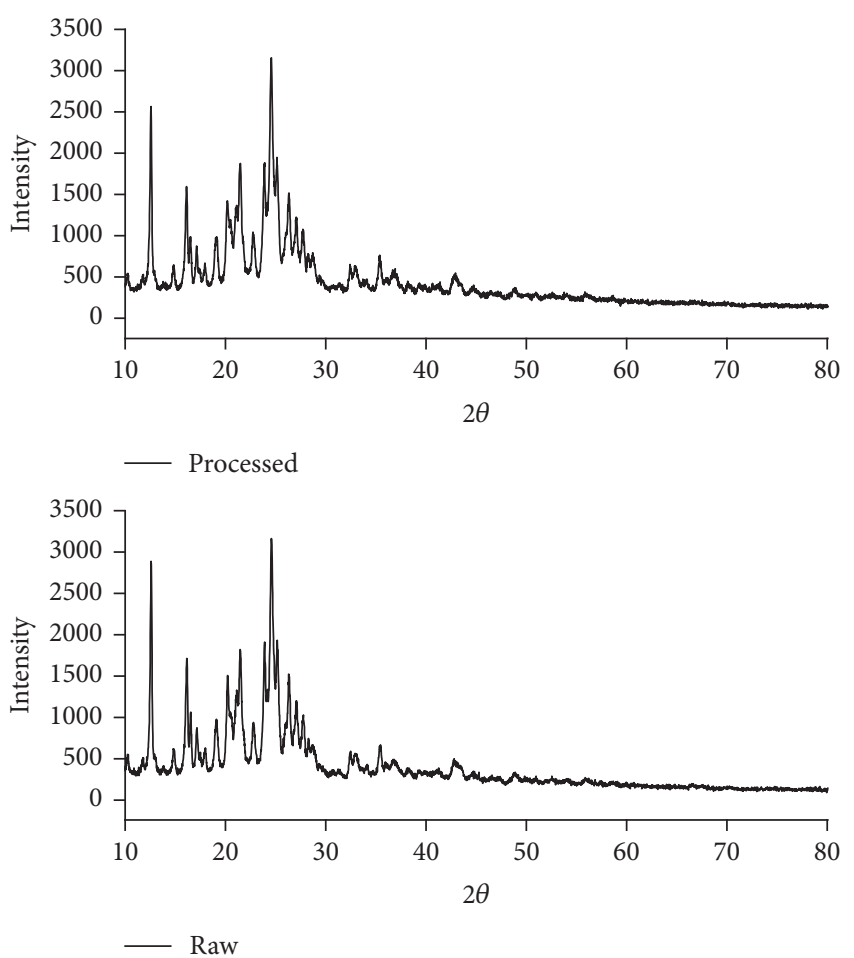

Figure 7: X-ray diffraction patterns of raw and processed cefquinome.

\section{Conclusions}

Annular gap nozzle was used in SEDS process to prepare cefquinome controlled size submicron particles. The optimal operating condition and univariate effect of every factor on the process were studied by orthogonal experiments. The effects of the four factors on particle size were revealed, that is, the concentration of solution, feeding speed of solution, precipitation pressure, and temperature, ranking first to forth. The optimal operating conditions for preparing cefquinome particles in SEDS process using annular gap nozzle were that solution concentration was $100 \mathrm{mg} / \mathrm{mL}$; the feeding speed was $9 \mathrm{~mL} / \mathrm{min}$; precipitation pressure was $10 \mathrm{MPa}$; and precipitation temperature was $316 \mathrm{~K}$. Confirmatory experiment showed that $D_{50}$ of processed cefquinome in optimal operating conditions was $0.73 \mu \mathrm{m}$. Univariate effect analysis showed that the cefquinome particle size increased with the increase of concentration of the solution or precipitation pressure but decreased with the increase of solution feeding speed. When precipitation temperature increased, the cefquinome particle size showed highest point and then decreased. Moreover, characterization of processed cefquinome particles was analyzed by SEM, FT-IR, and XRD. Analysis results indicated that the surface appearance of processed cefquinome particles was flakes. The chemical structure of processed cefquinome particles was not changed, and the crystallinity of processed cefquinome particles was lower than that of raw cefquinome particles. The application of annular gap nozzle would be beneficial to industrial applications of SEDS process. The process optimization of preparing controlled size submicron particles by SEDS process would be helpful for cefquinome to enhance efficacy and application in more fields.

\section{Conflicts of Interest}

The authors declare that there are no conflicts of interest regarding the publication of this paper.

\section{Acknowledgments}

This work was financially supported by Science and Technology Development Plan of Shandong Province: Supercritical Fluid Dyeing Technology Research and Device Development (2014GGX108001).

\section{References}

[1] J. M. Swinkels, T. J. G. M. Lam, M. J. Green, and A. J. Bradley, "Effect of extended cefquinome treatment on clinical persistence or recurrence of environmental clinical mastitis," Veterinary Journal, vol. 197, no. 3, pp. 682-687, 2013.

[2] V. Dinakaran, V. K. Dumka, B. Ranjan, R. Balaje, and P. K. Sidhu, "Pharmacokinetics following intravenous administration and pharmacodynamics of cefquinome in buffalo calves," Tropical Animal Health and Production, vol. 45, no. 7, pp. 1509-1512, 2013.

[3] K. G. Hopper, H. Leclair, and B. R. McCord, "A novel method for analysis of explosives residue by simultaneous detection of anions and cations via capillary zone electrophoresis," Talanta, vol. 67, no. 2, pp. 304-312, 2005.

[4] M. Henczka, J. Bałdyga, and B. Y. Shekunov, "Particle formation by turbulent mixing with supercritical antisolvent," Chemical Engineering Science, vol. 60, no. 8-9, pp. 2193-2201, 2005.

[5] X. H. Zhao, X. Q. Chen, Y. G. Zu, R. Jiang, and D. M. Zhao, "Recrystallization and micronization of taxol using the supercritical antisolvent (SAS) process," Industrial and Engineering Chemistry Research, vol. 51, no. 28, pp. 9591-9597, 2012.

[6] S. D. Javeer, R. Patole, and P. Amin, "Enhanced solubility and dissolution of simvastatin by HPMC-based solid dispersions prepared by hot melt extrusion and spray-drying method," Journal of Pharmaceutical Investigation, vol. 43, no. 6, pp. 471480, 2013.

[7] J. Xu, "Preparation of ultra-fine clarithromycin particles by antisolvent recrystallization," Key Engineering Materials, vol. 680, pp. 534-537, 2016.

[8] M. Trotta, M. Gallarate, F. Pattarino, and S. Morel, "Emulsions containing partially water-miscible solvents for the preparation of drug nanosuspensions," Journal of Controlled Release, vol. 76, no. 1-2, pp. 119-128, 2001.

[9] P. Sheth, H. Sandhu, D. Singhal, W. Malick, N. Shah, and M. S. Kislalioglu, "Nanoparticles in the pharmaceutical industry and the use of supercritical fluid technologies for nanoparticle production," Current Drug Delivery, vol. 9, no. 3, pp. 269-284, 2012.

[10] Y. B. Jiang, W. L. Sun, and W. Wang, "Recrystallization and micronization of 10-hydroxycamptothecin by supercritical antisolvent process," Industrial \& Engineering Chemistry Research, vol. 51, no. 6, pp. 2596-2602, 2012.

[11] Y. Miyazaki, H. Sugihara, A. Nishiura, K. Kadota, Y. Tozuka, and H. Takeuchi, "Application of combinational supercritical CO2 techniques to the preparation of inhalable particles," Journal of Drug Delivery Science and Technology, vol. 36, pp. 1-9, 2016. 
[12] B. Y. Shekunov, J. Baldyga, and P. York, "Particle formation by mixing with supercritical antisolvent at high Reynolds numbers," Chemical Engineering Science, vol. 56, no. 7, pp. 2421-2433, 2001.

[13] T. Yan, Y. Cheng, Z. Wang, D. Huang, H. Miao, and Y. Zhang, "Preparation and characterization of baicalein powder micronized by the SEDS process," Journal of Supercritical Fluids, 2015.

[14] E. Reverchon, I. De Marco, and G. Della Porta, "Tailoring of nano- and micro-particles of some superconductor precursors by supercritical antisolvent precipitation," Journal of Supercritical Fluids, vol. 23, no. 1, pp. 81-87, 2002.

[15] J. Bałdyga, D. Kubicki, B. Y. Shekunov, and K. B. Smith, "Mixing effects on particle formation in supercritical fluids," Chemical Engineering Research and Design, vol. 88, no. 9, pp. 1131-1141, 2010.

[16] R. Ghaderi, P. Artursson, and J. Carlfors, "Preparation of biodegradable microparticles using solution- enhanced dispersion by supercritical fluids (SEDS)," Pharmaceutical Research, vol. 16, no. 5, pp. 676-681, 1999.

[17] D. J. Jarmer, C. S. Lengsfeld, and T. W. Randolph, "Manipulation of particle size distribution of poly(L-lactic acid) nanoparticles with a jet-swirl nozzle during precipitation with a compressed antisolvent," Journal of Supercritical Fluids, vol. 27, no. 3, pp. 317336, 2003.

[18] X. Kefeng, W. Weiqiang, H. Dedong, H. Zhihui, Q. Yanpeng, and L. Yan, "Preparation of cefquinome nanoparticles by using the supercritical antisolvent process," Journal of Nanomaterials, vol. 2015, Article ID 767945, 2015.

[19] W. Z. He, Q. L. Suo, Z. H. Jiang, A. Shan, and H. L. Hong, "Precipitation of ephedrine by SEDS process using a specially designed prefilming atomizer," The Journal of Supercritical Fluids, vol. 31, no. 1, pp. 101-110, 2004.

[20] W. Weiqiang, Y. Liu, and K. Xiao, "An internal mixing nozzle for preparing nanomaterials by supercritical fluid method," $C N$, 200510042540, vol. 7, pp. 2005-3, 2005.

[21] W. Li, G. Liu, L. Li, J. Wu, Y. Lü, and Y. Jiang, "Effect of process parameters on co-precipitation of paclitaxel and poly(L-lactic Acid) by supercritical antisolvent process," Chinese Journal of Chemical Engineering, vol. 20, no. 4, pp. 803-813, 2012.

[22] J. Baldyga, "Mixing and fluid dynamics effects in particle precipitation processes," KONA Powder and Particle Journal, vol. 2016, no. 33, pp. 127-149, 2016.

[23] C. Chang, A Fundamental Study on the Preparation of Drugloaded Polymer Microparticles by the Supercritical Fluid Technology, Dalian University of Technology, 2012.

[24] J. Bałdyga, R. Czarnocki, B. Y. Shekunov, and K. B. Smith, "Particle formation in supercritical fluids-Scale-up problem," Chemical Engineering Research and Design, vol. 88, no. 3, pp. 331-341, 2010.

[25] V. Majerik, G. Charbit, E. Badens et al., "Bioavailability enhancement of an active substance by supercritical antisolvent precipitation," Journal of Supercritical Fluids, vol. 40, no. 1, pp. 101-110, 2007. 


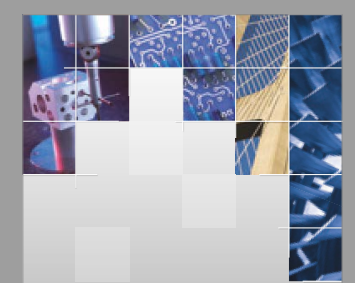

\section{Enfincering}
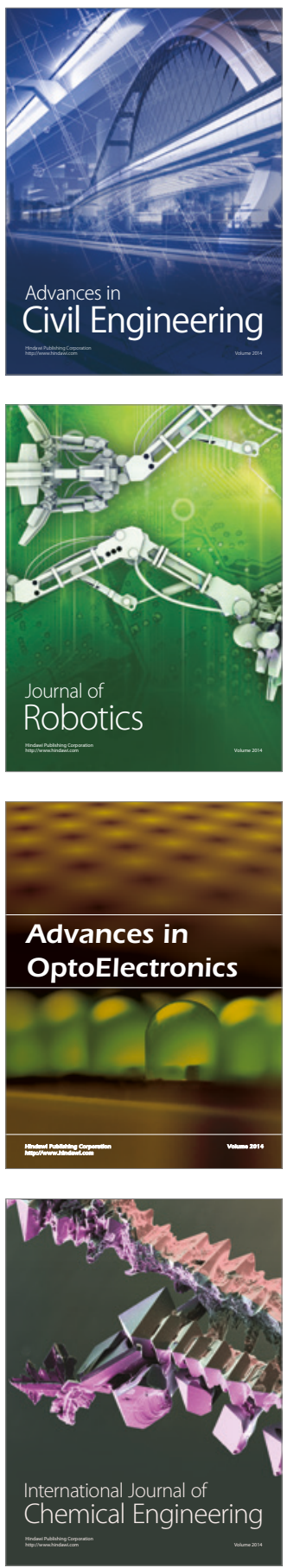

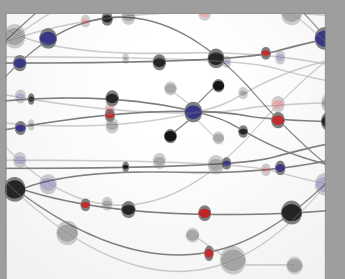

The Scientific World Journal

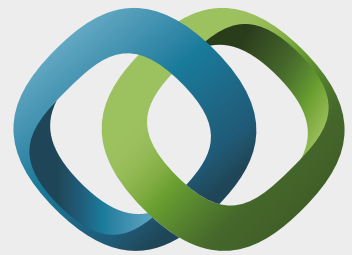

\section{Hindawi}

Submit your manuscripts at

https://www.hindawi.com
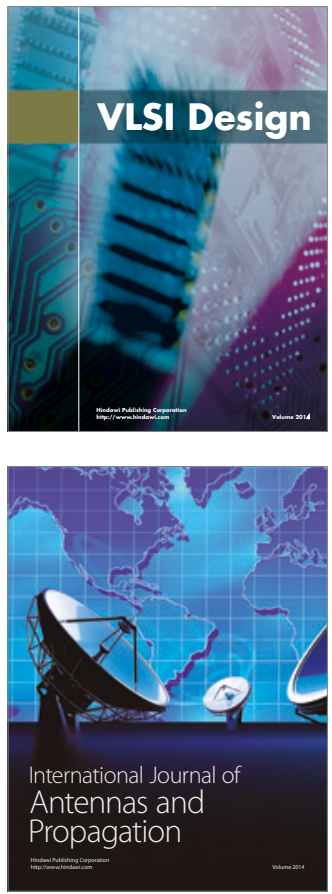

\section{Rotating}

Machinery
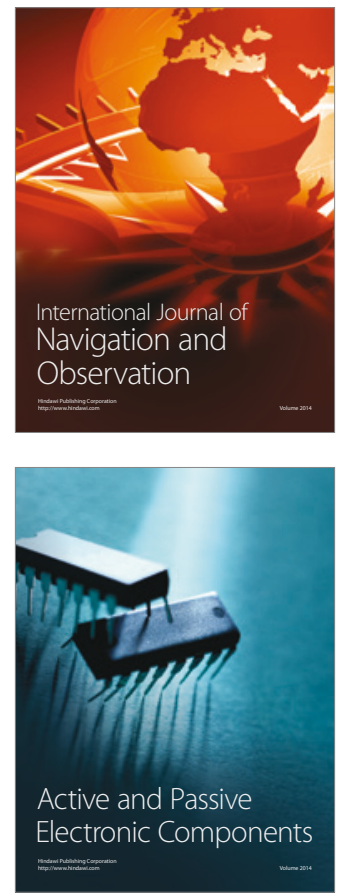
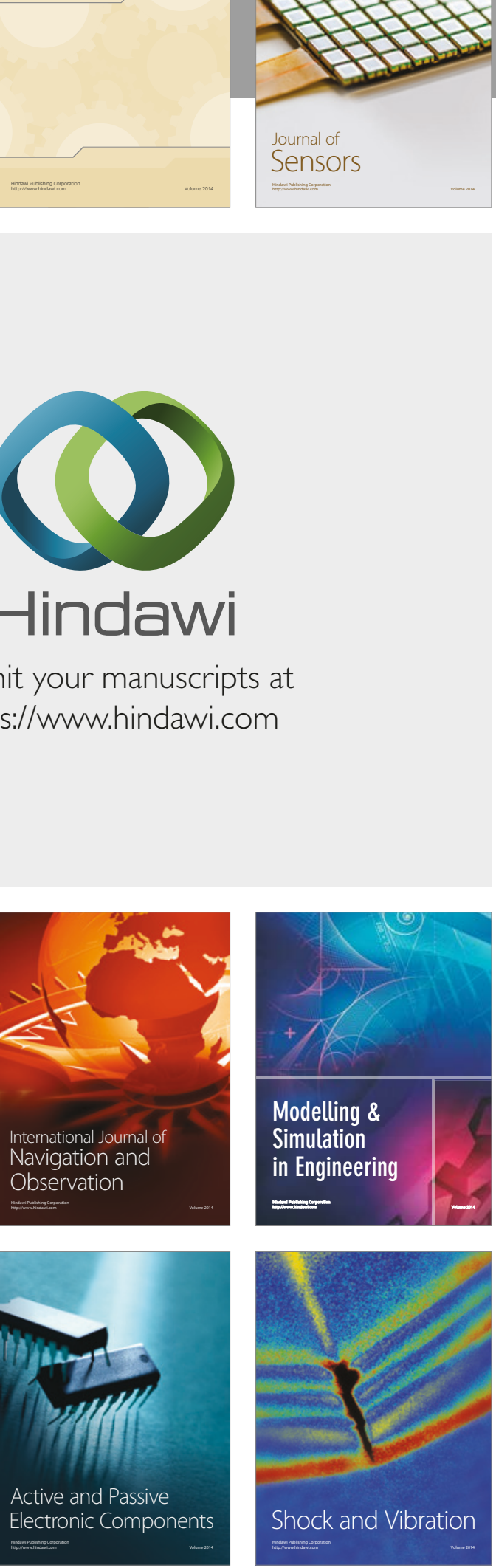
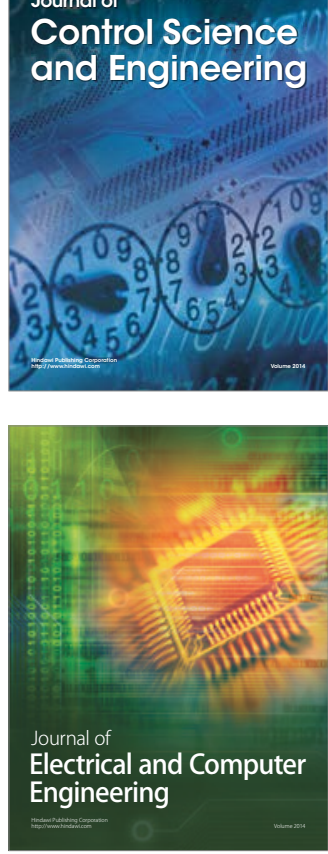

Distributed

Journal of

Control Science

and Engineering
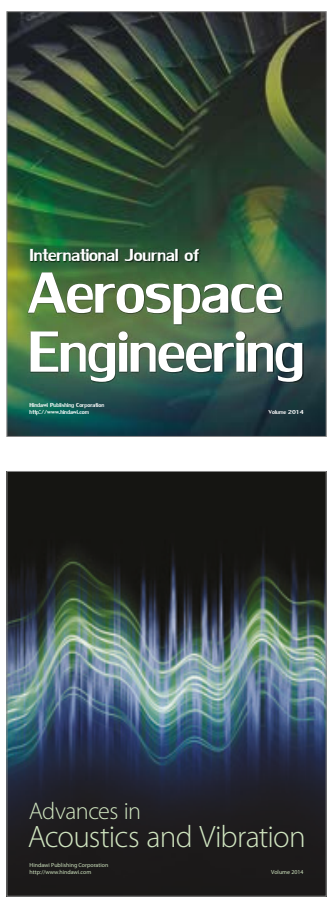

Sensor Networks 